\title{
Context and Barriers to the Prescription of Nonoccupational Postexposure Prophylaxis Among HIV Medical Care Providers: National Internet-Based Observational Study in China
}

Haibo Ding ${ }^{1,2,3,4^{*}}, \mathrm{PhD} ;$ Zehao Ye $\mathrm{e}^{1,2,3,4^{*}}, \mathrm{MPH}$; Weiming Tang ${ }^{5}, \mathrm{PhD} ;$ Xiaojie Huang ${ }^{6}, \mathrm{PhD}$; Hui Wang ${ }^{7}, \mathrm{PhD}$; Sitong $\mathrm{Cui}^{1,2,3,4}, \mathrm{MPH}$; Yongjun Jiang ${ }^{1,2,3,4}, \mathrm{PhD}$; Wenqing Geng ${ }^{1,2,3,4}, \mathrm{PhD}$; Junjie $\mathrm{Xu}^{1,2,3,4 *}, \mathrm{PhD}$; Hong Shang ${ }^{1,2,3,4^{*}}, \mathrm{PhD}$

\footnotetext{
${ }^{1}$ National Health Commission Key Laboratory of AIDS Immunology (China Medical University), National Clinical Research Center for Laboratory Medicine, The First Affiliated Hospital of China Medical University, Shenyang, Liaoning Province, China

${ }^{2}$ Key Laboratory of AIDS Immunology, Chinese Academy of Medical Sciences, Shenyang, China

${ }^{3}$ Key Laboratory of AIDS Immunology of Liaoning Province, Shenyang, China

${ }^{4}$ Collaborative Innovation Center for Diagnosis and Treatment of Infectious Diseases, Hangzhou, China

${ }^{5}$ Project-China, University of North Carolina at Chapel Hill, Guangzhou, China

${ }^{6}$ Center for Infectious Diseases, Beijing You'an Hospital, Capital Medical University, Beijing, China

${ }^{7}$ Shenzhen Third People's Hospital, Shenzhen, China

*these authors contributed equally
}

\section{Corresponding Author:}

Hong Shang, PhD

National Health Commission Key Laboratory of AIDS Immunology (China Medical University)

National Clinical Research Center for Laboratory Medicine

The First Affiliated Hospital of China Medical University

No 155, Nanjing North Street, Heping District

Shenyang, Liaoning Province, 110001

China

Phone: 8683282634

Email: hongshang100@hotmail.com

\section{Abstract}

Background: Nonoccupational postexposure prophylaxis (nPEP) is an effective HIV biomedical prevention strategy. The research and use of nPEP are mainly concentrated in the developed world, while little is known about the knowledge, attitudes, and practices of nPEP among HIV medical care providers in developing countries.

Objective: We aimed to assess the nPEP knowledge and prescribing practice among HIV medical care providers in mainland China.

Methods: HIV medical care providers were recruited in China during May and June 2019 through an online survey regarding nPEP-related knowledge, attitudes, and clinical prescription experiences. Multivariable logistic regression was performed to identify factors associated with prescribing nPEP among HIV medical care providers.

Results: A total of 777 eligible participants participated in this study from 133 cities in 31 provinces in China. Of the participants, $60.2 \%$ (468/777) were unfamiliar with nPEP and only 53.3\% (414/777) of participants ever prescribed nPEP. HIV care providers who worked in a specialized infectious disease hospital (vs general hospital, adjusted odds ratio [aOR] 2.49; 95\% CI 1.85-3.37), had practiced for 6-10 years (vs 5 or fewer years, aOR 3.28; 95\% CI 2.23-4.80), had practiced for 11 years or more (vs 5 or fewer years, aOR 3.75; 95\% CI 2.59-5.45), and had previously prescribed occupational PEP (oPEP, aOR 4.90; 95\% CI 3.29-7.29) had a significantly positive association with prescribing nPEP. However, unfamiliarity with nPEP (aOR 0.08; 95\% CI 0.05-0.11), believing nPEP may promote HIV high-risk behavior (aOR 0.53; 95\% CI 0.36-0.77) or result in HIV drug resistance (aOR 0.53; 95\% CI 0.36-0.77) among key populations, and self-reported having no written oPEP guideline in place (aOR 0.53 ; 95\% CI $0.35-0.79)$ were negatively associated with nPEP prescription behavior.

Conclusions: HIV medical care providers have insufficient nPEP knowledge and an inadequate proportion of prescribing, which may impede the scale-up of nPEP services to curb HIV acquisition. The implementation of tailored nPEP training or retraining to HIV medical care providers would improve this situation. 
(JMIR Public Health Surveill 2021;7(3):e24234) doi: 10.2196/24234

\section{KEYWORDS}

WeChat; nonoccupational postexposure prophylaxis (nPEP); HIV medical care providers; training; key populations; internet; China; social media; barrier; prophylaxis

\section{Introduction}

\section{HIV Epidemic in Key Populations}

The Joint United Nations Program on HIV/AIDS (UNAIDS) and World Health Organization (WHO) estimate that 38 million people were living with HIV in 2019, with over two-thirds concentrated in low-income developing countries $[1,2]$. The epidemic of HIV is concentrated in key populations [3], including men who have sex with men (MSM) [4,5]. There has been an increasing number of new HIV infections in China over the past 5 years [6], with approximately 958,000 people reported living with HIV in 2019 [7]. Data based on the HIV Sentinel Surveillance System in China showed that MSM had an HIV infection prevalence rate of $6.9 \%$ in 2018 [8].

\section{Effectiveness of Nonoccupational Postexposure Prophylaxis}

Nonoccupational postexposure prophylaxis (nPEP) is an effective and cost-effective HIV biomedical prevention strategy $[9,10]$. There have been no randomized controlled trials for nPEP due to ethical considerations, but a case-control study of occupational postexposure prophylaxis (oPEP) demonstrated an $81 \%$ reduction in the odds of HIV transmission [11]. nPEP guidelines have been in use by WHO, European AIDS Clinical Society, United States, and Canada for years to offer guidance on nPEP uptake [12-16], and the research and use on nPEP in the developed world is extensive. However, nPEP services are not widely used in most developing countries with relatively severe HIV epidemics, even though some have released their own guidelines. Additional efforts are needed to target nPEP uptake to end the AIDS epidemic by 2030 .

\section{Previous Studies and Existing Gap}

HIV medical care providers play an indispensable role in nPEP uptake, especially medication prescription [17]. Previous surveys have reported on HIV care providers prescribing nPEP in developed countries [18-23], most often including factors such as practice specialty, the number of persons living with HIV in treatment, provider familiarity with nPEP, and the nPEP guideline in place $[18,19,23]$. As these surveys were conducted in developed countries with nPEP guidelines, it is uncertain whether the situation is similar in developing countries without nPEP guidelines. A clear understanding of obstacles encountered by providers in developing countries without nPEP guidelines will be beneficial to the scale-up of nPEP uptake and control of the HIV epidemic.

A positive attitude has emerged recently in China on the use of nPEP for HIV prevention. The Chinese Center for Disease Control and Prevention (China CDC) carried out a pilot program of nPEP among MSM in 7 provinces to promote the uptake of PEP and preexposure prophylaxis (PrEP) between 2018 and 2019 [24]. In addition, China released the Program to Reduce
AIDS (2019-2020) to ensure that the HIV epidemic was controlled at a low level, which encouraged the application of nPEP programs [25]. Considering an increasing body of evidence, China released the nPEP guideline in October 2020 [26]; however, little is known about the knowledge, attitude, and practice of nPEP in HIV medical care providers in China. It is necessary to understand the nPEP perception among HIV medical care providers and barriers associated with prescribing nPEP to provide targeted interventions.

\section{Objectives}

We sought to understand nPEP perceptions and practice among HIV medical care providers and factors correlated with nPEP prescription under the current efforts of scale-up of nPEP services.

\section{Methods}

\section{Study Design and Participant Enrollment}

We conducted a nationwide online survey among HIV medical care providers during May and June 2019. After a presurvey to adjust the questionnaire items, a survey invitation was sent to 937 HIV medical care providers from two WeChat groups, "National clinicians group majors in HIV/AIDS" and "National physician platform for communicating of difficult cases in HIV/AIDS." These WeChat groups are currently the leading online WeChat-based communication platforms for HIV-related clinicians in China, with the largest number of registered HIV-related clinicians. The investigator released recruitment information via the WeChat groups, including the study aims, procedure, and requirements of the survey. Eligible participants completed an anonymous online survey by scanning the QR (quick response) code link of the online questionnaire. Inclusion criteria included being age 18 years or older, self-reported practicing in HIV-related medical institutions, having treated at least one person living with HIV over the past year, and providing online informed consent to the study content and protocol. Each individual was allowed to access the online survey once. Each internet protocol address is restricted to answer only one questionnaire. A 30-yuan honorarium (approximately US \$4.50) was paid to each participant through WeChat accounts after completion of the 5 to 10 minute questionnaire survey. We used contact information only for releasing rewards and did not disclose it to others.

\section{Data Collection}

After providing informed consent, participants completed anonymous online questionnaires on sociodemographic characteristics (age, sex, ethnicity, and educational background), hospital types, technical titles, practice specialty, length of practice, nPEP-related knowledge, attitudes, and clinical prescription experiences (Multimedia Appendix 1). The 3 questions on nPEP-related knowledge (with possible answers 
yes, no, and I don't know) were as follows: Do you think China has issued national clinical guidelines on nPEP? Do you think unprotected anal intercourse (UAI) risk exceeds percutaneous occupational exposure risk? Do you think percutaneous occupational exposure risk exceeds unprotected vaginal intercourse (UVI) exposure risk?

Data on nPEP-related attitudes (with possible answers agree, neutral, and disagree) were also collected as follows: Do you agree that clinicians have enough time to prescribe nPEP? Do you agree that prescribing nPEP in clinical settings is feasible? Do you agree that prescribing nPEP will promote HIV drug resistance? Do you agree that prescribing nPEP will promote high-risk behaviors?

Additionally, we collected nPEP-related experiences, including the experience of encountering key populations seeking nPEP help and nPEP prescribing history. Before submission, participants could review all items of the questionnaire and make sure mandatory items were completed. To evaluate the impact of geographic HIV epidemic level on prescribing nPEP, we categorized regions into high, middle, and low epidemic levels according to the number of HIV/AIDS cases reported in 2017 (Multimedia Appendix 2). The top one-third of regions were classified as having a high epidemic level, while the bottom one-third were classified as having a low epidemic level. Further, to evaluate the impact of the nPEP pilot program recently conducted by China $\mathrm{CDC}$, we divided the provinces into 2 categories, nPEP and non-nPEP pilot provinces. The study protocol was reviewed and approved by the institutional review board committee of the First Affiliated Hospital of China Medical University ([2019]2015-138-9). We have completed the Checklist for Reporting Results of Internet E-Surveys (CHERRIES) for this study (Multimedia Appendix 3).

\section{Sample Size Calculation}

We calculated the sample size of participants based on the formula of a 2-sided confidence interval for one proportion: $\mathrm{N}$ $=\mathrm{Z}^{2}{ }_{1-\alpha / 2} \times \mathrm{P} \times(1-\mathrm{P}) / \mathrm{D}^{2}$. For a conservative estimate of sample size, the proportion of nPEP prescription $(\mathrm{P})$ was set to be 0.5 .
At a 5\% significance level ( $\alpha$ ) and 5\% margin of error (D), the smallest sample size was calculated as 384 observations.

\section{Data Analysis}

Category variables were described by frequency and percentage and continuous variables by mean and standard deviation or median and interquartile range (IQR). All the core variables involved in the questionnaire are required. For variables with a missing ratio of less than 5\%, we imputed related missing values in the database by mean for continuous variables and mode for categorical variables in the course of data processing. Variables with more than $5 \%$ missing ratio would have been deleted, but there were none in this study. For the needs of analysis, we transformed some variables (eg, familiarity of nPEP) into the binary forms yes (extremely familiar, very familiar) or no (generally familiar, not familiar very much, not familiar at all). We used univariable logistic regression to calculate odds ratios (OR) and their $95 \%$ confidence intervals for factors associated with prescribing nPEP among HIV medical care providers. Multivariable logistic regression was applied to estimate associations between predictors and nPEP prescribing history after adjustment for age, sex, ethnicity, and educational background. We used SPSS Statistics version 26.0 (IBM Corporation) for analysis. Variables with 2-tailed $P<.05$ were considered statistically significant.

\section{Results}

\section{Demographic Characteristics}

Of the HIV medical care providers reached, 82.9\% (777/937) of eligible participants from 133 cities in the 31 provinces of China participated in this study (Figure 1A). Participants had a median age of 42 (IQR 36-48) years. A majority of participants were female (417/777, 53.7\%), of Han ethnicity (712/777, $91.6 \%)$, had undergraduate or above level of education (743/777, $95.6 \%)$, had been in practice for more than 5 years $(432 / 777$, $55.6 \%$ ), and had a technical title of attending physician or above (695/777, 89.4\%). Approximately half $(394 / 777,50.7 \%)$ of participants worked at specialized hospitals for infectious diseases (Table 1). 
Figure 1. Distribution of participants (A), proportion of nonoccupational postexposure prophylaxis (nPEP) prescriptions (B), HIV epidemic level (C), and nPEP pilot versus nonpilot provinces of 31 total in China (D).

A

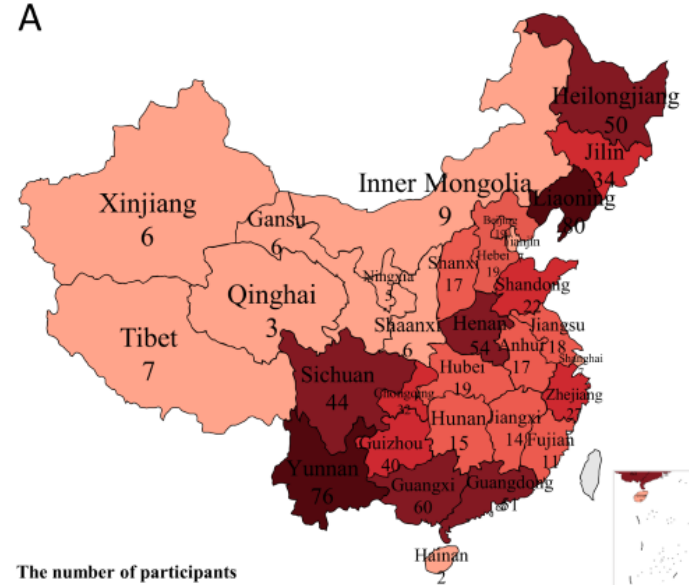

The number of participants

C

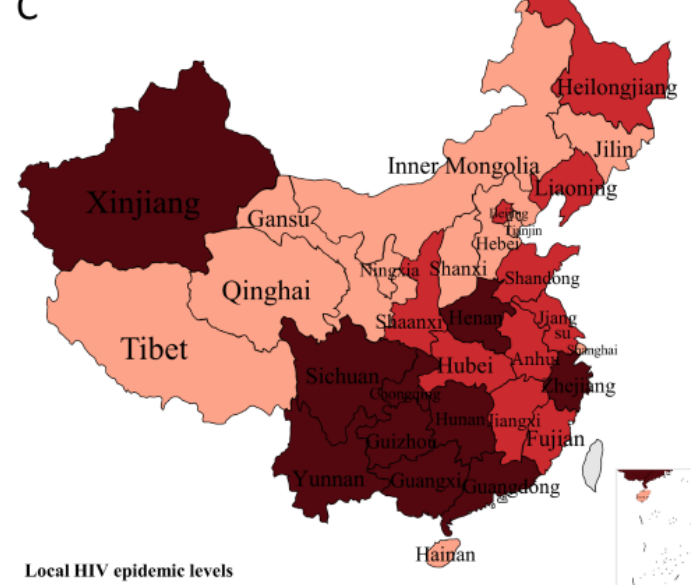

Low Middle $\mathbf{H i g h}$ Not available
B

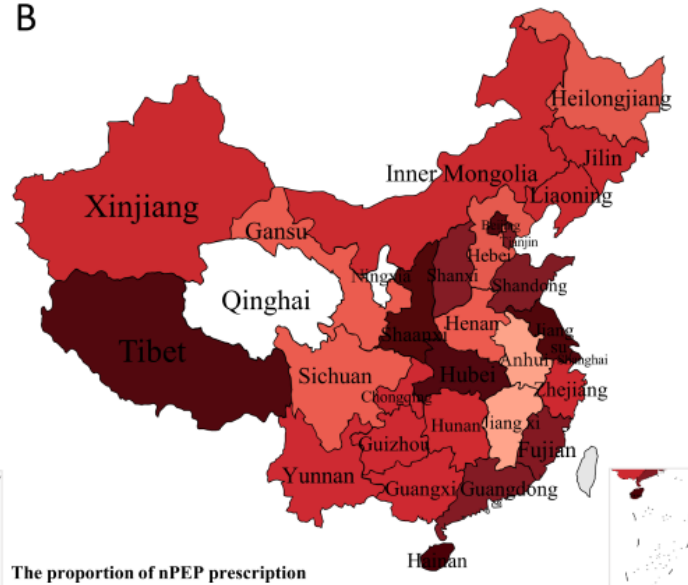

$D^{0}$

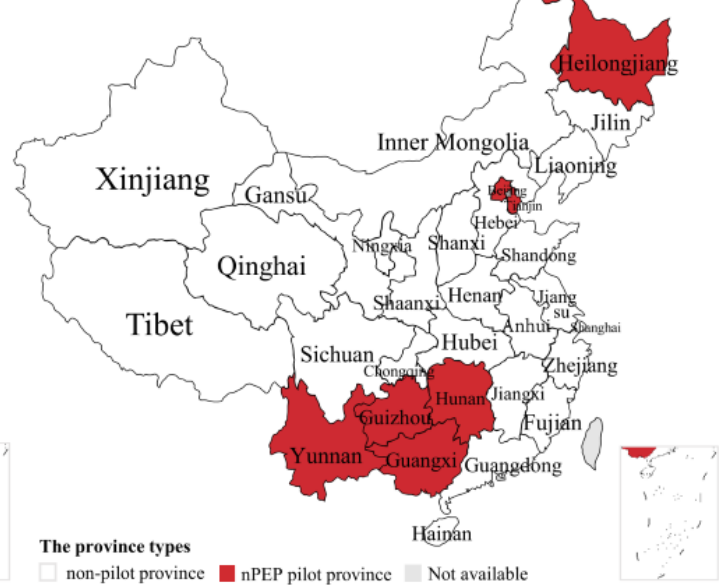


Table 1. Demographics and knowledge of HIV medical care providers in China ( $\mathrm{n}=777)$.

\begin{tabular}{|c|c|}
\hline Variable & Total, n (\%) \\
\hline \multicolumn{2}{|l|}{ Age in years } \\
\hline$\leq 25$ & $3(0.4)$ \\
\hline $26-39$ & $302(38.9)$ \\
\hline $40-49$ & $331(42.6)$ \\
\hline $50-59$ & $131(16.9)$ \\
\hline$\geq 60$ & $10(1.3)$ \\
\hline \multicolumn{2}{|l|}{ Ethnicity } \\
\hline Han & $712(91.6)$ \\
\hline Non-Han & $65(8.4)$ \\
\hline \multicolumn{2}{|l|}{ Sex } \\
\hline Male & $360(46.3)$ \\
\hline Female & $417(53.7)$ \\
\hline \multicolumn{2}{|l|}{ Educational background } \\
\hline High school/technical secondary school & $5(0.6)$ \\
\hline Junior college & $29(3.7)$ \\
\hline Undergraduate or above & $743(95.6)$ \\
\hline \multicolumn{2}{|l|}{ Administrative regions of China } \\
\hline North & $71(9.1)$ \\
\hline Northeast & $164(21.1)$ \\
\hline East & $116(14.9)$ \\
\hline South Central & $201(25.9)$ \\
\hline Southwest & $199(25.6)$ \\
\hline Northwest & $26(3.3)$ \\
\hline \multicolumn{2}{|l|}{ nPEP $^{\mathrm{a}}$ pilot program province ${ }^{\mathrm{b}}$} \\
\hline Yes & $267(34.4)$ \\
\hline No & $510(65.6)$ \\
\hline \multicolumn{2}{|l|}{ Local HIV epidemic level $^{c}$} \\
\hline Low & $116(14.9)$ \\
\hline Middle & $256(32.9)$ \\
\hline High & $405(52.1)$ \\
\hline \multicolumn{2}{|l|}{ Hospital type } \\
\hline Specialized hospital for infectious diseases & $394(50.7)$ \\
\hline General hospital & $383(49.3)$ \\
\hline \multicolumn{2}{|l|}{ Technical title } \\
\hline General physician & $82(10.6)$ \\
\hline Attending physician & $274(35.3)$ \\
\hline Associate chief physician & $221(28.4)$ \\
\hline Chief physician & $200(25.7)$ \\
\hline \multicolumn{2}{|l|}{ Clinical practice specialty } \\
\hline HIV care professional & $649(83.5)$ \\
\hline Non-HIV care professional & $128(16.5)$ \\
\hline
\end{tabular}




\begin{tabular}{|c|c|}
\hline Variable & Total, n (\%) \\
\hline \multicolumn{2}{|c|}{ Practice time in years } \\
\hline$\leq 5$ & $345(44.4)$ \\
\hline $6-10$ & $184(23.7)$ \\
\hline$\geq 11$ & $248(31.9)$ \\
\hline \multicolumn{2}{|c|}{ Unfamiliar with oPEP ${ }^{d}$} \\
\hline Yes & $292(37.6)$ \\
\hline No & $485(62.4)$ \\
\hline \multicolumn{2}{|c|}{ Unfamiliar with nPEP } \\
\hline Yes & $468(60.2)$ \\
\hline No & $309(39.8)$ \\
\hline \multicolumn{2}{|c|}{ Do you think China has issued a national clinical guideline on nPEP? } \\
\hline Yes & $551(70.9)$ \\
\hline No & $97(12.5)$ \\
\hline I don't know & $129(16.6)$ \\
\hline \multicolumn{2}{|c|}{ Do you think UAI ${ }^{\mathrm{e}}$ exposure risk between males exceeds percutaneous occupational exposure risk? } \\
\hline Yes & $667(85.8)$ \\
\hline No & $61(7.9)$ \\
\hline I don't know & $49(6.3)$ \\
\hline \multicolumn{2}{|c|}{ Do you think percutaneous occupational exposure risk exceeds UVI ${ }^{f}$ exposure risk? } \\
\hline Yes & $532(68.5)$ \\
\hline No & $199(25.6)$ \\
\hline I don't know & $46(5.9)$ \\
\hline
\end{tabular}

a $\mathrm{nPEP}$ : nonoccupational postexposure prophylaxis.

${ }^{b}$ PEP pilot programs were conducted by China CDC in provinces Yunnan, Beijing, Tianjin, Heilongjiang, Hunan, Guangxi, and Guizhou.

${ }^{\mathrm{c}}$ Local HIV epidemic level was categorized according to the number of HIV/AIDS cases reported in 2017.

doPEP: occupational postexposure prophylaxis.

${ }^{\mathrm{e}} \mathrm{UAI}$ : unprotected anal intercourse.

${ }^{\mathrm{f}} \mathrm{UVI}$ : unprotected vaginal intercourse.

\section{Knowledge, Experiences, and Attitudes}

Overall, only 39.8\% (309/777) of participants reported that they were familiar with nPEP, and just 6.8\% (53/777) correctly answered all 3 nPEP knowledge-related questions (Table 1). Further, 59.3\% (461/777) of participants had provided medical services to fewer than 50 persons living with HIV over the past month, 40.2\% (312/777) reported that they had encountered key populations seeking nPEP prescriptions over the past 6 months, and $74.0 \%(575 / 777)$ reported that they had a written oPEP guideline in place (Table 2). Among providers from Northwest China, 69.2\% (18/26) were unfamiliar with nPEP and only $23.1 \%(6 / 26)$ had provided HIV care to more than 50 persons living with HIV over the past month.
***A survey of participant opinions on the most suitable population for nPEP prescriptions showed that most participants were inclined to prescribe nPEP to people having a partner living with HIV $(543 / 777,69.9 \%)$, people who had been sexually assaulted $(485 / 777,62.4 \%)$, as well as those with histories of sexually transmitted disease (483/777, 62.2\%), unprotected sexual intercourse $(466 / 777,60.0 \%)$, irregular visits to the clinic $(454 / 777,58.4 \%)$, drug injection $(452 / 777,58.2 \%)$, and poor drug adherence $(402 / 777,51.8 \%)$. Moreover, $58.9 \%$ (458/777) agreed that they had adequate time to prescribe nPEP, and $27.3 \%(212 / 777)$ and $32.9 \%$ (256/777) reported that nPEP may promote HIV drug resistance and HIV high-risk behavior, respectively, among key populations. The problems that primarily concerned participants were high drug cost (452/777, $58.2 \%$ ) and adverse effects of nPEP (438/777, 56.4\%; Table 2). 
Table 2. Experiences and attitudes among HIV medical care providers in China ( $\mathrm{n}=777)$.

\begin{tabular}{|c|c|}
\hline Variable & Total, n (\%) \\
\hline \multicolumn{2}{|l|}{ Number of persons living with HIV in treatment in the past month } \\
\hline$\leq 50$ & $461(59.3)$ \\
\hline$>50$ & $316(40.7)$ \\
\hline Ever prescribed nPEP ${ }^{\mathrm{a}}$ & $414(53.3)$ \\
\hline Ever prescribed oPEP ${ }^{b}$ & $197(25.4)$ \\
\hline Self-reported having a written oPEP guideline in place & $575(74.0)$ \\
\hline \multicolumn{2}{|l|}{ Key populations seeking nPEP help over the past 6 months } \\
\hline Often/occasionally (more than 1 per month) & $312(40.2)$ \\
\hline Never/rarely (less than 1 per month) & $465(59.8)$ \\
\hline Having barriers for prescribing nPEP in place & $236(30.4)$ \\
\hline \multicolumn{2}{|l|}{ nPEP-related attitudes } \\
\hline \multicolumn{2}{|l|}{ Have adequate time to prescribe nPEP } \\
\hline Agree & $458(58.9)$ \\
\hline Neutral & $235(30.2)$ \\
\hline Disagree & $84(10.8)$ \\
\hline \multicolumn{2}{|l|}{ nPEP will promote HIV drug resistance } \\
\hline Agree & $212(27.3)$ \\
\hline Neutral & $308(39.6)$ \\
\hline Disagree & $257(33.1)$ \\
\hline \multicolumn{2}{|l|}{ nPEP will promote HIV risky behavior } \\
\hline Agree & $256(32.9)$ \\
\hline Neutral & $302(38.9)$ \\
\hline Disagree & $219(28.2)$ \\
\hline \multicolumn{2}{|l|}{ Feasible to provide nPEP in place } \\
\hline Agree & $712(91.6)$ \\
\hline Neutral & $57(7.3)$ \\
\hline Disagree & $8(1.0)$ \\
\hline Worry about being blamed for prescribing nPEP due to no nPEP drug indication & $583(75.0)$ \\
\hline Necessary to have expert consensus for nPEP & $693(89.2)$ \\
\hline Necessary to establish outpatient for nPEP & $620(79.8)$ \\
\hline \multicolumn{2}{|l|}{ Concerns about prescribing nPEP } \\
\hline Increased risk behavior & $291(37.5)$ \\
\hline Poor medication adherence & $310(39.9)$ \\
\hline HIV drug resistance & $238(30.6)$ \\
\hline Side effects & $438(56.4)$ \\
\hline High cost & $452(58.2)$ \\
\hline No specific guidance for nPEP & $159(20.5)$ \\
\hline No nPEP drug indication & $126(16.2)$ \\
\hline Resources reduced for HIV-positive patients & $121(15.6)$ \\
\hline Other problems & $40(5.1)$ \\
\hline
\end{tabular}

\footnotetext{
${ }^{a}$ nPEP: nonoccupational postexposure prophylaxis.
} 
b oPEP: occupational postexposure prophylaxis.

\section{Factors Associated With Prescribing nPEP}

In total, $53.3 \%$ (414/777) of participants had previously prescribed nPEP, among which $38.9 \%$ (161/414) reported that they had experienced barriers during the process (Table 2). The proportion prescribing nPEP in each province ranged from $30 \%$ to $80 \%$ (Figure 1B; Multimedia Appendix 4). The proportions of participants having a history of nPEP prescription were $51.9 \%$ (60/414), 56.3\% (144/414), and 51.7\% (210/414) in provinces with high, middle, and low HIV epidemic levels, respectively (Figure 1C; Table 3), while in the $7 \mathrm{nPEP}$ pilot provinces, $56.2 \%$ $(150 / 267)$ of participants had a history of nPEP prescription (Figure 1D).

Table 3 presents the results of univariable and multivariable logistic regression analyses of factors associated with prescribing nPEP among HIV medical care providers. The forest plot of the results of multivariable logistic regression analysis can be found in Multimedia Appendix 5. After initial adjustment for age, sex, ethnicity, and educational background, we found providers from Northwest regions had a significantly lower proportion of nPEP prescription (vs North China; adjusted odds ratio [aOR] $0.35 ; 95 \% \mathrm{CI} 0.14-0.89$ ). We further adjusted age, sex, ethnicity, educational background, and administrative regions covariates, and independent factors positively associated with prescribing nPEP were as follows: practicing in a specialized infectious disease hospital (vs general hospital, aOR 2.49; 95\% CI 1.85-3.37), working professionally in HIV care (vs nonprofessional in HIV care, aOR 6.13; 95\% CI 3.83-9.81), having a technical title of chief physician (vs general physician, aOR 2.16; 95\% CI 1.15-4.05), having 6 to 10 years of practice (vs 5 or fewer years, aOR 3.28; 95\% CI 2.23-4.80), having 11 or more years of practice (vs 5 or fewer years, aOR $3.75 ; 95 \%$ CI 2.59-5.45), providing medical services to more than 50 persons living with HIV over the past month (vs 50 or fewer persons living with HIV, aOR 3.89; 95\% CI 2.83-5.36), and having previously prescribed oPEP (aOR 4.90, 95\% CI 3.29-7.29; each $P<.05$ ). In contrast, unfamiliar with oPEP (aOR 0.12 ; $95 \%$ CI 0.08-0.16), unfamiliar with nPEP (aOR 0.08; $95 \%$ CI 0.05-0.11), unaware that risks of UAI exceed percutaneous occupational exposure risk (aOR 0.63; 95\% CI 0.42-0.95); self-reported having no written oPEP guideline in place (aOR 0.53 ; $95 \% \mathrm{CI} 0.35-0.79$ ), and believing that $\mathrm{nPEP}$ may promote HIV high-risk behavior (aOR 0.53 ; 95\% CI 0.36-0.77) or result in HIV drug resistance (aOR 0.53; 95\% CI 0.36-0.77) among key populations were negatively associated with nPEP prescription behavior (each $P<.05$ ). However, practicing in provinces with high HIV epidemic level and nPEP pilot programs were not significantly associated with nPEP prescription behavior. 
Table 3. Univariable and multivariable logistic regression analyses of associations of potential predictors and nPEP prescription history among HIV medical care providers in China $(\mathrm{n}=777)$.

\begin{tabular}{lllll}
\hline Variable & nPEP prescription & Crude model & Adjusted model $P$ value \\
\hline Yes, $\mathrm{n}(\%)$ & No $(\mathrm{n}, \%)$ & $\mathrm{OR}^{\mathrm{b}}(95 \% \mathrm{CI})$ & $\mathrm{aOR}^{\mathrm{c}}(95 \% \mathrm{CI})$
\end{tabular}

Age in years

$<40$
$40-49$
$\geq 50$

Ethnicity

$$
\text { Han }
$$

Non-Han

Sex

$$
\begin{aligned}
& \text { Female } \\
& \text { Male }
\end{aligned}
$$

\section{Educational background}

Undergraduate or above
Junior college

High school/technical secondary school

\section{Administrative regions of China}

North
Northeast
East
South Central
Southwest
Northwest

nPEP pilot province ${ }^{f}$

No
Yes

Local HIV epidemic level ${ }^{\mathrm{g}}$

Low

High

\section{Hospital type}

General hospital

Specialized hospital for infectious diseases

\section{Technical title}

General physician

Attending physician

Associate chief physician

Chief physician

\section{Clinical practice specialty}

$\begin{array}{ll}153(50.2) & 152(49.8) \\ 179(54.1) & 152(45.9) \\ 82(58.2) & 59(41.8)\end{array}$

$\operatorname{Ref}^{\mathrm{d}}$

$1.17(0.86-1.60)$

$1.38(0.92-2.07)$

$\begin{array}{lll}374(52.5) & 338(47.5) & \text { Ref } \\ 40(61.5) & 25(38.5) & 1.45(0.86-2.43)\end{array}$

$233(55.9) \quad 184(44.1)$

$181(50.3) \quad 179(49.7)$

Ref

$0.80(0.60-1.06)$

$\begin{array}{lll}400(53.8) & 343(46.2) & \text { Ref } \\ 13(44.8) & 16(55.2) & 0.61(0.33-1.47) \\ 1(20.0) & 4(80.0) & 0.21(0.02-1.93)\end{array}$

$45(63.4) \quad 26(36.6) \quad$ Ref

76 (46.3)

$88(53.7)$

Ref

63 (54.3)

53 (45.7)

$0.50(0.28-0.88)$

$0.69(0.38-1.26)$

113 (56.2)

$88(43.8)$

$0.74(0.43-1.30)$

107 (53.8)

$92(46.2)$

$0.67(0.39-1.17)$

$10(38.5)$

$16(61.5)$

$0.36(0.14-0.91)$

-

$264(51.8)$

$246(48.2)$

Ref

$150(56.2)$

$117(43.8)$

$1.20(0.89-1.61)$

Ref

$1.13(0.82-1.58)$

Ref

$0.51(0.28-0.91)$

-
-

-
.02
.27
.49
.22
.03

$0.71(0.38-1.31) \quad .27$

$0.82(0.46-1.45) \quad .49$

$0.70(0.40-1.23) \quad .22$

$0.35(0.14-0.89)$

60 (51.7)

$56(48.3)$

Ref

$144(56.3)$

$112(43.8)$

$1.20(0.77-1.86)$

$210(51.9)$

$195(48.1)$

$1.01(0.67-1.52)$

Ref

$1.45(0.85-2.49)$

$0.50(0.24-1.03)$

$\begin{array}{lllll}158(41.3) & 225(58.7) & \text { Ref } & \text { Ref } & - \\ 256(65.0) & 138(35.0) & 2.64(1.98-3.53) & 2.49(1.85-3.37) & <.001\end{array}$

$\begin{array}{lllll}38(46.3) & 44(53.7) & \text { Ref } & \text { Ref } & - \\ 141(51.5) & 133(48.5) & 1.23(0.75-2.01) & 1.25(0.75-2.07) & .40 \\ 105(47.5) & 116(52.5) & 1.05(0.63-1.74) & 0.98(0.55-1.77) & .96 \\ 130(65.0) & 70(35.0) & 2.15(1.28-3.63) & 2.16(1.15-4.05) & .02\end{array}$

$26(20.3) \quad 102(79.7) \quad$ Ref $\quad$ Ref

$388(59.8) \quad 261(40.2) \quad 5.83(3.69-9.22)$
-
-

-
-
.02
.27
.49
.22
.03

.17

.06
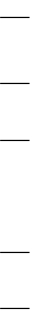

001
$6.13(3.83-9.81)$

$<.001$

Non-HIV care professional

HIV care professional 


\begin{tabular}{|c|c|c|c|c|c|}
\hline Variable & \multicolumn{2}{|c|}{$n P E P^{a}$ prescription } & Crude model & Adjusted model & $P$ value \\
\hline \multicolumn{6}{|l|}{ Practice time in years } \\
\hline$\leq 5$ & $128(37.1)$ & $217(62.9)$ & Ref & Ref & - \\
\hline $6-10$ & $118(64.1)$ & $66(35.9)$ & $3.03(2.09-4.40)$ & $3.28(2.23-4.80)$ & $<.001$ \\
\hline$\geq 11$ & $168(67.7)$ & $80(32.3)$ & $3.56(2.52-5.02)$ & $3.75(2.59-5.45)$ & $<.001$ \\
\hline \multicolumn{6}{|l|}{ Unfamiliar with oPEP ${ }^{h}$} \\
\hline No & $345(71.1)$ & $140(28.9)$ & Ref & Ref & - \\
\hline Yes & $69(23.6)$ & $223(76.4)$ & $0.13(0.09-0.18)$ & $0.12(0.08-0.16)$ & $<.001$ \\
\hline \multicolumn{6}{|l|}{ Unfamiliar with nPEP } \\
\hline No & $264(85.4)$ & 45 (14.6) & Ref & Ref & - \\
\hline Yes & $150(32.1)$ & $318(67.9)$ & $0.08(0.06-0.12)$ & $0.08(0.05-0.11)$ & $<.001$ \\
\hline \multicolumn{6}{|c|}{ China has issued a national clinical guideline on nPEP } \\
\hline No & $115(50.9)$ & $111(49.1)$ & Ref & Ref & - \\
\hline Yes/ I don't know & $299(54.3)$ & $252(45.7)$ & $0.04(0.01-0.11)$ & $0.03(0.01-0.09)$ & $<.001$ \\
\hline \multicolumn{6}{|c|}{ UAI ${ }^{\mathrm{i}}$ risk between males exceeds percutaneous occupational exposure risk } \\
\hline Yes & $366(54.9)$ & $301(45.1)$ & Ref & Ref & - \\
\hline No/I don't know & $48(43.6)$ & $62(56.4)$ & $0.64(0.42-0.96)$ & $0.63(0.42-0.95)$ & .03 \\
\hline \multicolumn{6}{|c|}{ Percutaneous occupational exposure risk exceeds $\mathrm{UVI}^{\mathrm{j}}$ exposure risk } \\
\hline Yes & $238(44.7)$ & $294(55.3)$ & Ref & Ref & - \\
\hline No/I don’t know & $176(71.8)$ & $69(28.2)$ & $3.15(2.27-4.37)$ & $3.27(2.33-4.58)$ & $<.001$ \\
\hline \multicolumn{6}{|c|}{ Number of persons living with HIV in treatment in the past month } \\
\hline$\leq 50$ & $184(39.9)$ & $277(60.1)$ & Ref & Ref & - \\
\hline$>50$ & $230(72.8)$ & $86(27.2)$ & $4.03(2.95-5.49)$ & $3.89(2.83-5.36)$ & $<.001$ \\
\hline \multicolumn{6}{|l|}{ Ever prescribed oPEP } \\
\hline No & $264(45.5)$ & $316(54.5)$ & Ref & Ref & - \\
\hline Yes & $150(76.1)$ & $47(23.9)$ & $3.82(2.65-5.51)$ & $4.90(3.29-7.29)$ & $<.001$ \\
\hline \multicolumn{6}{|c|}{ Self-reported having a written oPEP guideline in place } \\
\hline Yes & $350(60.9)$ & $225(39.1)$ & Ref & Ref & - \\
\hline No & $56(45.9)$ & $66(54.1)$ & $0.55(0.37-0.81)$ & $0.53(0.35-0.79)$ & .002 \\
\hline Unsure & $8(10.0)$ & $72(90.0)$ & $0.07(0.03-0.15)$ & $0.07(0.03-0.15)$ & $<.001$ \\
\hline \multicolumn{6}{|c|}{ Key populations seeking nPEP over the past 6 months } \\
\hline Never/rarely (<1/month) & $149(32.0)$ & $316(68.0)$ & Ref & Ref & - \\
\hline Often/occasionally (>1/month) & $265(84.9)$ & $47(15.1)$ & $11.96(8.29-17.25)$ & $13.86(9.42-20.39)$ & $<.001$ \\
\hline \multicolumn{6}{|l|}{ nPEP-related attitudes } \\
\hline \multicolumn{6}{|c|}{ Adequate time to prescribe nPEP } \\
\hline Disagree & $44(52.4)$ & $40(47.6)$ & Ref & Ref & - \\
\hline Neutral & $109(46.4)$ & $126(53.6)$ & $0.79(0.48-1.30)$ & $0.83(0.50-1.38)$ & .47 \\
\hline Agree & $261(57.0)$ & $197(43.0)$ & $1.20(0.76-1.92)$ & $1.24(0.77-2.00)$ & .38 \\
\hline \multicolumn{6}{|c|}{ Feasible to provide nPEP in place } \\
\hline Disagree & $3(37.5)$ & $5(62.5)$ & Ref & Ref & - \\
\hline Neutral & $11(19.3)$ & $46(80.7)$ & $0.40(0.08-1.93)$ & $0.42(0.09-2.09)$ & .29 \\
\hline Agree & $400(56.2)$ & $312(43.8)$ & $2.14(0.51-9.01)$ & $2.20(0.51-9.51)$ & .29 \\
\hline
\end{tabular}




\begin{tabular}{|c|c|c|c|c|c|}
\hline \multirow{2}{*}{$\begin{aligned} & \text { Variable } \\
& \text { Disagree }\end{aligned}$} & \multicolumn{2}{|c|}{$\mathrm{nPEP}^{\mathrm{a}}$ prescription } & \multirow{2}{*}{$\begin{array}{l}\text { Crude model } \\
\text { Ref }\end{array}$} & \multirow{2}{*}{$\begin{array}{l}\text { Adjusted model } \\
\text { Ref }\end{array}$} & \multirow{2}{*}{$P$ value } \\
\hline & $161(62.6)$ & $96(37.4)$ & & & \\
\hline Neutral & $157(51.0)$ & $151(49.0)$ & $0.62(0.44-0.87)$ & $0.65(0.46-0.92)$ & .014 \\
\hline Agree & $96(45.3)$ & $116(54.7)$ & $0.49(0.34-0.72)$ & $0.53(0.36-0.77)$ & .001 \\
\hline \multicolumn{6}{|c|}{ nPEP will promote HIV risky behavior } \\
\hline Disagree & $137(62.6)$ & $82(37.4)$ & Ref & Ref & - \\
\hline Neutral & $161(53.3)$ & $141(46.7)$ & $0.68(0.48-0.98)$ & $0.69(0.48-0.99)$ & .04 \\
\hline Agree & $116(45.3)$ & $140(54.7)$ & $0.50(0.34-0.72)$ & $0.53(0.36-0.77)$ & .001 \\
\hline \multicolumn{6}{|c|}{ Concern about prescribing nPEP } \\
\hline No & $13(54.2)$ & $11(45.8)$ & Ref & Ref & - \\
\hline Yes & $401(53.3)$ & $352(46.7)$ & $0.96(0.43-2.18)$ & $0.97(0.43-2.23)$ & .95 \\
\hline \multicolumn{6}{|c|}{ Concern about promoting HIV high-risk behaviors } \\
\hline No & $268(55.1)$ & $218(44.9)$ & Ref & Ref & - \\
\hline Yes & $146(50.2)$ & $145(49.8)$ & $0.82(0.61-1.10)$ & $0.83(0.61-1.11)$ & .21 \\
\hline \multicolumn{6}{|c|}{ Concern about poor adherence to $\mathrm{nPEP}$} \\
\hline No & $261(55.9)$ & $206(44.1)$ & Ref & Ref & - \\
\hline Yes & $153(49.4)$ & $157(50.6)$ & $0.77(0.58-1.03)$ & $0.81(0.60-1.09)$ & .16 \\
\hline \multicolumn{6}{|c|}{ Concern about HIV drug resistance } \\
\hline No & $288(53.4)$ & $251(46.6)$ & Ref & Ref & - \\
\hline Yes & $126(52.9)$ & $112(47.1)$ & $0.98(0.72-1.33)$ & $0.97(0.71-1.32)$ & .83 \\
\hline \multicolumn{6}{|c|}{ Concern about side effects of drugs } \\
\hline No & $181(53.4)$ & $158(46.6)$ & Ref & Ref & - \\
\hline Yes & $233(53.2)$ & $205(46.8)$ & $0.99(0.75-1.32)$ & $0.98(0.73-1.31)$ & .88 \\
\hline \multicolumn{6}{|c|}{ Concern about cost of nPEP } \\
\hline No & $158(48.6)$ & $167(51.4)$ & Ref & Ref & - \\
\hline Yes & $256(56.6)$ & $196(43.4)$ & $1.38(1.04-1.84)$ & $1.39(1.03-1.86)$ & .03 \\
\hline \multicolumn{6}{|c|}{ Concern about lack of nPEP clinical guideline } \\
\hline No & $326(52.8)$ & $292(47.2)$ & Ref & Ref & - \\
\hline Yes & $88(55.3)$ & $71(44.7)$ & $1.11(0.78-1.58)$ & $1.19(0.83-1.71)$ & .34 \\
\hline \multicolumn{6}{|c|}{ Concern about lack of drug indications } \\
\hline No & $338(51.9)$ & $313(48.1)$ & Ref & Ref & - \\
\hline Yes & $76(60.3)$ & $50(39.7)$ & $1.41(0.95-2.08)$ & $1.50(1.01-2.24)$ & .045 \\
\hline \multicolumn{6}{|c|}{ Concern about reducing treatment resources of HIV-positive patients } \\
\hline No & $377(57.5)$ & $279(42.5)$ & Ref & Ref & - \\
\hline Yes & $37(30.6)$ & $84(69.4)$ & $0.33(0.22-0.50)$ & $0.32(0.21-0.49)$ & $<.001$ \\
\hline
\end{tabular}

${ }_{\text {a }}$ PEP: nonoccupational postexposure prophylaxis.

${ }^{\mathrm{b}} \mathrm{OR}$ : odds ratio.

${ }^{\mathrm{c}} \mathrm{aOR}$ : adjusted odds ratio.

${ }^{\mathrm{d}}$ Ref: reference.

${ }^{\mathrm{e}} \mathrm{N} / \mathrm{A}$ : not applicable.

$\mathrm{f}_{\mathrm{nPEP}}$ pilot programs were conducted by China CDC in provinces Yunnan, Beijing, Tianjin, Heilongjiang, Hunan, Guangxi, and Guizhou.

${ }^{\mathrm{g}}$ Local HIV epidemic level was categorized according to the number of HIV/AIDS cases reported in 2017.

hoPEP: occupational postexposure prophylaxis.

${ }^{\mathrm{i}} \mathrm{UAI}$ : unprotected anal intercourse.

${ }^{\mathrm{j}} \mathrm{UVI}$ : unprotected vaginal intercourse. 


\section{Discussion}

\section{Principal Findings and Significance}

Our study showed that most HIV medical care providers in China were unfamiliar with nPEP, and only a bit more than half of participants had previously prescribed nPEP. We also found that unfamiliarity with nPEP, self-report of having no written PEP-related guideline in place, and less HIV care experience were possibly important barriers to nPEP prescription among HIV medical care providers. This study addresses a gap in the research and shows the negative impact of insufficient knowledge, such as misunderstanding nPEP-related HIV drug resistance and side effects, on the scale-up of nPEP services and subsequent inadequate nPEP prescription by clinicians. It may help public health policymakers learn about HIV medical provider perception of nPEP, thereby providing the opportunity to implement corresponding measures to counter nPEP-related obstacles. Our data also have great significance for further practice after initiating the national nPEP guideline to inform HIV medical care providers in their implementation of nPEP. Additionally, the results of this study are a reference for other countries with similar HIV contexts and insufficient uptake of nPEP services.

\section{Comparison With Prior Work}

We found that the proportion $(60.2 \%)$ of HIV care providers unfamiliar with nPEP was higher than that reported in a previous study from the United States (51.5\%) [19]. About 70\% of participants incorrectly thought China had already issued a national clinical guideline on nPEP before this survey. This finding may indicate that a high proportion of HIV medical care providers confused oPEP guidelines, released in 2004 [27], with nPEP guidelines or thought the Chinese Guidelines for Diagnosis and Treatment of HIV/AIDS, updated in 2018 [28], were nPEP guidelines. An accordingly high proportion of prescribing nPEP, though, was not found among these providers. This can be attributed to insufficient familiarity with nPEP because of a lack of media advertisements and tailored training. Newly reported HIV cases in China still show an increasing trend [6], however, with strong acceptance of and great demand for nPEP among key populations [29]. This gap could hinder efforts to curb the spread of the HIV epidemic; therefore, intensified publicity through diverse channels and reinforced training or retraining should be offered to improve the knowledge of these providers.

In our study, the proportion of lifetime prescribing of nPEP among HIV care providers (53.3\%) was lower than that reported by previous studies from the United States $(67.1 \%)$ [23], France $(58.0 \%)$ [30], and Spain $(77.3 \%)$ [31], which may indicate a huge gap between China and developed countries in the prevention of HIV spread. The gap between the proportion of HIV medical care providers prescribing nPEP and the demand of key populations for nPEP [29] implies that improving the level of nPEP prescription would likely have a remarkable effect on preventing HIV spread. Previous studies found knowledge plays an indispensable role in PrEP prescription behavior $[32,33]$. Another study found that HIV-related training has a significant correlation with the increased nPEP and PrEP knowledge and the improved PrEP prescribing practice among HIV care providers [20], which also means a possible effect on nPEP prescription through increasing nPEP knowledge by training. Furthermore, there are many nPEP-related challenges, including risk assessment and management of viral hepatitis, frequent transitions from $\mathrm{nPEP}$ to PrEP, and the management of low follow-up rates and poor medication adherence [34], which, if addressed improperly, will bring adverse effects and even harm from nPEP. These challenges will not be resolved in the near-term without targeted nPEP training integrating practical skills exercises into didactic sessions, thereby ultimately delaying the progression of HIV prevention.

\section{Factors Associated With nPEP Prescription}

In addition, we identified independent factors positively correlated with nPEP prescription among HIV medical care providers. Compared with providers working in general hospitals, those in specialized infectious disease hospitals had a significantly higher proportion of prescribing nPEP, probably due to more awareness of HIV-related information. HIV-related stigma remains severe in China, however, and key populations are more inclined to visit the general hospital for HIV-related services to protect their privacy and avoid disclosure [35], which may limit access to nPEP services. Thus, for those providers in general hospitals, reinforced targeted training is necessary to improve their perception and enhance willingness to prescribe $n P E P$. We also found significantly higher proportions of nPEP prescription among HIV care professionals (vs non-HIV care professionals), chief physicians (vs general physicians), providers with more than 5 years of working experience (vs 5 or fewer years), and those having provided HIV care to more than 50 persons living with HIV over the past month (vs 50 or more persons living with HIV). Providers with professional knowledge, high-ranking technical titles, and rich HIV care experience are usually skilled, which can attract more patients. They as well have more opportunities to attend HIV-related international conferences and obtain information on nPEP from other countries. This finding suggests that nPEP-related training should also be focused on young providers to enrich their nPEP knowledge and improve practical skills, which could even be delivered during student medical training. Moreover, the establishment of specific support mechanisms via senior clinicians would help overcome the obstacles to prescribe nPEP faced by young clinicians.

In contrast, we found that unfamiliarity with nPEP, incorrect beliefs that nPEP will promote HIV drug resistance or high-risk behaviors, self-reported lack of written oPEP guideline in working settings, and unfamiliarity with oPEP were all negatively correlated with nPEP prescription among HIV medical care providers. Although the nPEP guideline was released in October 2020 [26], further outreach efforts to clinicians in working settings are needed or existing incorrect perceptions caused by insufficient nPEP knowledge will continue to impede the scale-up of nPEP services. The oPEP guideline was released about 15 years ago, so HIV medical care providers are more familiar with oPEP $(62.4 \%)$ than nPEP $(39.8 \%)$. 
The associations between the practice of oPEP and nPEP, two methods targeted at different types of HIV exposure, have rarely been explored in previous studies. In our study, the level of prescribing nPEP was higher among HIV medical care providers who had previously prescribed oPEP than that among those who had not. There are many similar features between nPEP and oPEP about assessing HIV exposure risk, principles of treatment, and the types of antiviral drugs. HIV medical care providers who master the oPEP practice may be relatively more familiar with prescribing nPEP. Therefore, training programs combining nPEP with oPEP can create a synergistic effect on both prescription behaviors of HIV care providers. Notably, despite the emphasis of simplifying prescribing practice from the updated WHO guideline for nPEP [13], regardless of HIV exposure types, different types vary in the risk of acquiring HIV and subsequent laboratory test items [36]. Providers confusing the standards of nPEP and oPEP practices may well prescribe nPEP improperly to some individuals at low risk of HIV acquisition [37] or miss some items, such as pregnancy testing and the collection of forensic specimens [36]. It again underlines the necessity to provide targeted nPEP training or retraining based on the nPEP guideline.

Compared with North China (63.4\%), we found a surprisingly lower proportion of nPEP prescription in Northwest $(38.5 \%)$ and Northeast $(46.3 \%$ ) regions where providers have insufficient nPEP familiarity (30.8\% and $33.5 \%$, respectively) and less HIV care practice $(23.1 \%$ and $32.3 \%$, respectively). In the contrast, the HIV epidemic is highly prevalent in Xinjiang Province, located in Northwest China. Hence, more attention should be paid to these regions, especially the Northwest with its limited resources, in future national nPEP training efforts. Given the difficulty of organizing centralized training for HIV medical care providers from various regions, internet-based online training is critical for nPEP implementation. It has clear advantages for transmitting up-to-date knowledge and ideas, particularly for providers in the Northwest regions with insufficient resources for nPEP implementation. Besides traditional didactic sessions, online simulation trainings related to practical skills are also promising methods to offset the gap of resource from regions.

Finally, there was no significant association between high HIV epidemic level and nPEP prescription behavior of HIV medical care providers. This indicates that key populations in those provinces at high HIV epidemic level may miss the opportunity to obtain nPEP services even after exposure to HIV. Similarly, we did not find the effect of an nPEP pilot program on nPEP prescription behavior of these providers, which may be explained by the relative short duration implementation time and limited number of involved cities. Therefore, it is necessary to further enhance the advertisement of nPEP at a national level to raise the wide attention of HIV medical care providers.

\section{Strengths and Limitations}

Our study has many strengths. First, this is a representative cross-sectional study of nPEP perception and prescribing practice among HIV medical care providers in all 31 provinces of China, and the sources of participants from previous studies have been limited. Second, the sample size of this study was larger than those of previous similar studies. Last, as this is the first study of nPEP perception and prescribing practice among HIV medical care providers in China, the results represent a vital reference that could contribute to solving the obstacles to nPEP prescription, popularizing the use of nPEP nationwide, and controlling HIV spread among key populations.

This study also has limitations. First, this study was conducted by two WeChat groups, and our results rely on self-reporting data, which to some extent would cause sampling bias and reporting bias. Second, HIV care providers from Hong Kong, Macao, and Taiwan were not included in the WeChat groups, and the number of samples from western China (ie, Tibet) was insufficient; hence, the results may not well represent the characteristics of HIV medical care providers from these regions. Additionally, given the cross-sectional design, the causal relationships between prescribing nPEP and other factors are uncertain and will require further prospective studies to confirm.

\section{Conclusions}

This is the first cross-sectional survey of nPEP-related knowledge, attitudes, and prescribing experience among HIV medical care providers in a country without extensive use of nPEP services. Our results underline the insufficient nPEP knowledge and inadequate proportion of nPEP prescription among these providers. Implementing targeted nPEP training or retraining through the internet, particularly for young providers from general hospitals, should be priorities to eliminate obstacles in popularizing nPEP services and ultimately reducing HIV incidence among national key populations.

\section{Acknowledgments}

JX and HD conceived and designed the study. HD collected and cleaned data. JX and ZY analyzed the data and interpreted results. JX and ZY wrote and revised the manuscript. HD and ZY contributed equally as first authors. JX and HS contributed equally as corresponding authors. All authors have read and approved the final manuscript as submitted. The authors thank staff and all clinicians who participated in this study for their efforts. This work was supported by the National Natural Science Foundation of China (81872674), the Mega-Projects of national science research (13th Five-Year Plan [2017ZX10201101-002-007]), and National Science and Technology Major Project (2018ZX10101-001-001-003).

\section{Conflicts of Interest}

None declared. 


\section{Multimedia Appendix 1}

English version of the questionnaire.

[DOCX File, 26 KB-Multimedia Appendix 1]

\section{Multimedia Appendix 2}

Number of HIV/AIDS cases reported in the 31 provinces of China in 2017.

[XLS File (Microsoft Excel File), 33 KB-Multimedia Appendix 2]

\section{Multimedia Appendix 3}

Checklist for Reporting Results of Internet E-Surveys.

[DOC File , 64 KB-Multimedia Appendix 3]

\section{Multimedia Appendix 4}

Proportion of nPEP prescriptions in 31 provinces in China.

[XLS File (Microsoft Excel File), 30 KB-Multimedia Appendix 4]

\section{Multimedia Appendix 5}

Forest plot of the results of multivariable logistic regression analysis.

[DOC File, 110 KB-Multimedia Appendix 5]

\section{References}

1. People living with HIV and new HIV infections. The Joint United Nations Programme on HIV/AIDS (UNAIDS). 2019.

URL: http://aidsinfo.unaids.org/ [accessed 2020-08-06]

2. Global Health Observatory (GHO) data. World Health Organization (WHO). 2018. URL: https://www.who.int/gho/hiv/en/ [accessed 2020-08-06]

3. The Gap Report, 2014. UNAIDS. URL: https://www.unaids.org/sites/default/files/media asset/UNAIDS Gap report en. pdf [accessed 2020-08-06]

4. Beyrer C, Baral SD, Walker D, Wirtz AL, Johns B, Sifakis F. The expanding epidemics of HIV type 1 among men who have sex with men in low- and middle-income countries: diversity and consistency. Epidemiol Rev 2010;32:137-151. [doi: 10.1093/epirev/mxq011] [Medline: 20573756]

5. Beyrer C, Sullivan P, Sanchez J, Baral SD, Collins C, Wirtz AL, et al. The increase in global HIV epidemics in MSM. AIDS 2013 Nov 13;27(17):2665-2678. [doi: 10.1097/01.aids.0000432449.30239.fe] [Medline: 23842129]

6. Database for HIV/AIDS. Chinese Center for Disease Control and Prevention. URL: http://www.phsciencedata.cn/Share/ ky sjml.jsp?id=c2ca694e-3995-4c7f-9078-3ed0aaf14556 [accessed 2020-08-06]

7. The progress of HIV prevention. The National Health Commission of the People's Republic of China. 2019. URL: http:/ /www.nhc.gov.cn/jkj/s3586/201911/c2388ce70bdd404ea6dfcd886591784d.shtml [accessed 2020-08-06]

8. Key Population Atlas. UNAIDS. 2018. URL: https://kpatlas.unaids.org/dashboard [accessed 2020-07-29]

9. Bryant J, Baxter L, Hird S. Non-occupational postexposure prophylaxis for HIV: a systematic review. Health Technol Assess 2009 Feb;13(14):1-60 [FREE Full text] [doi: 10.3310/hta13140] [Medline: 19236820]

10. Pinkerton SD, Martin JN, Roland ME, Katz MH, Coates TJ, Kahn JO. Cost-effectiveness of HIV postexposure prophylaxis following sexual or injection drug exposure in 96 metropolitan areas in the United States. AIDS 2004 Oct 21;18(15):2065-2073. [doi: 10.1097/00002030-200410210-00011] [Medline: 15577628]

11. Cardo DM, Culver DH, Ciesielski CA, Srivastava PU, Marcus R, Abiteboul D, et al. A case-control study of HIV seroconversion in health care workers after percutaneous exposure. Centers for Disease Control and Prevention Needlestick Surveillance Group. N Engl J Med 1997 Nov 20;337(21):1485-1490. [doi: 10.1056/NEJM199711203372101] [Medline: 9366579]

12. Smith DK, Grohskopf LA, Black RJ, Auerbach JD, Veronese F, Struble KA, U.S. Department of HealthHuman Services. Antiretroviral postexposure prophylaxis after sexual, injection-drug use, or other nonoccupational exposure to HIV in the United States: recommendations from the U.S. Department of Health and Human Services. MMWR Recomm Rep 2005 Jan 21;54(RR-2):1-20 [FREE Full text] [Medline: 15660015]

13. Guidelines on post-exposure prophylaxis for HIV and the use of co-trimoxazole prophylaxis for HIV-related infections among adults, adolescents and children: recommendations for a public health approach. World Health Organization. 2014. URL: https://apps.who.int/iris/bitstream/handle/10665/145719/9789241508193 eng. pdf;jsessionid=DF4A1858CDE737192782254FFE89DD9F?sequence=1 [accessed 2020-08-06]

14. EACS Guidelines. The European ADIS Clinical Society (EACS). 2019. URL: https://www.eacsociety.org/files/guidelines-10.

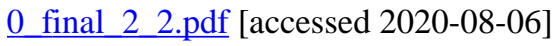


15. Centers for Disease Control and Prevention. Announcement: updated guidelines for antiretroviral postexposure prophylaxis after sexual, injection-drug use, or other nonoccupational exposure to HIV-United States, 2016. MMWR Morb Mortal Wkly Rep 2016 May 06;65(17):458 [FREE Full text] [doi: 10.15585/mmwr.mm6517a5] [Medline: 27149423]

16. Tan DHS, Hull MW, Yoong D, Tremblay C, O'Byrne P, Thomas R, Biomedical HIV Prevention Working Group of the CIHR Canadian HIV Trials Network. Canadian guideline on HIV pre-exposure prophylaxis and nonoccupational postexposure prophylaxis. Can Med Assoc J 2017 Nov 27;189(47):E1448-E1458 [FREE Full text] [doi: 10.1503/cmaj.170494] [Medline: 29180384]

17. Updated recommendations on first-line and second-line antiretroviral regimens and post-exposure prophylaxis and recommendations on early infant diagnosis of HIV: Interim guidance. World Health Organization. 2018. URL: https://apps. who.int/iris/bitstream/handle/10665/277395/WHO-CDS-HIV-18.51-eng.pdf?ua=1 [accessed 2020-07-31]

18. Rodríguez AE, Castel AD, Parish CL, Willis S, Feaster DJ, Kharfen M, et al. HIV medical providers' perceptions of the use of antiretroviral therapy as nonoccupational postexposure prophylaxis in 2 major metropolitan areas. J Acquir Immune Defic Syndr 2013 Nov 01;64 Suppl 1:S68-S79 [FREE Full text] [doi: 10.1097/QAI.0b013e3182a901a2] [Medline: 24126450]

19. Henny KD, Duke CC, Buchacz K, Brooks JT, Samandari T, Sutton MY. HIV prescriptions on the frontlines: primary care providers' use of antiretrovirals for prevention in the Southeast United States, 2017. Prev Med 2020 Jan;130:105875 [FREE Full text] [doi: 10.1016/j.ypmed.2019.105875] [Medline: 31678174$]$

20. Henny KD, Duke CC, Geter A, Gaul Z, Frazier C, Peterson J, et al. HIV-related training and correlates of knowledge, HIV screening and prescribing of nPEP and PrEP among primary care providers in southeast United States, 2017. AIDS Behav 2019 Nov;23(11):2926-2935 [FREE Full text] [doi: 10.1007/s10461-019-02545-1] [Medline: 31172333]

21. Landovitz RJ, Combs KB, Currier JS. Availability of HIV postexposure prophylaxis services in Los Angeles County. Clin Infect Dis 2009 Jun 01;48(11):1624-1627 [FREE Full text] [doi: 10.1086/598976] [Medline: 19400685]

22. Rutland E, Sundaram S, Mani R. The awareness of postexposure prophylaxis for HIV infection following sexual exposure in emergency departments in a regional HIV network. Int J STD AIDS 2010 Sep;21(9):636-637. [doi:

10.1258/ijsa.2010.010152] [Medline: 21097736]

23. John SA, Quinn KG, Pleuhs B, Walsh JL, Petroll AE. HIV post-exposure prophylaxis (PEP) awareness and non-occupational PEP (nPEP) prescribing history among U.S. healthcare providers. AIDS Behav 2020 Nov;24(11):3124-3131. [doi: 10.1007/s10461-020-02866-6] [Medline: 32300991]

24. Chinese Center for Disease Control and Prevention. URL: http://www.chinacdc.cn/zxdt/201912/t20191211_207519.html [accessed 2020-08-06]

25. The program to restrain AIDS (2019-2020). The National Health Commission of the People's Republic of China. 2019. URL: http://www.nhc.gov.cn/jkj/s3586/201910/40e14fe4c54e41999a147f79558e5eb1.shtml [accessed 2020-08-06]

26. Chinese Center for Disease Control and Prevention. Guidelines for HIV post-exposure prophylaxis. 2020. URL: http:/ /ncaids.chinacdc.cn/zxzx/zxzx/202011/W020201116802422550750.pdf [accessed 2020-12-14]

27. Guidelines for the protection of medical personnel against occupational exposure to HIV. The National Health Commission of the People's Republic of China. 2004. URL: http://www.nhc.gov.cn/wjw/gfxwj/201304/ 588fcab93194457cb2cdf3f150b3faac.shtml [accessed 2020-08-06]

28. AIDS and Hepatitis C Professional Group, Society of Infectious Diseases, Chinese Medical Association, Chinese Center for Disease ControlPrevention. [Chinese guidelines for diagnosis and treatment of HIV/AIDS (2018)]. Zhonghua Nei Ke Za Zhi 2018 Dec 01;57(12):867-884. [doi: 10.3760/cma.j.issn.0578-1426.2018.12.002] [Medline: $\underline{\text { 30486555] }}$

29. Hou J, Wu Y, Xie L, Meng S, Fu R, Zheng H, et al. Post-exposure prophylaxis: an underutilized biomedical HIV prevention method among gay, bisexual and other men who have sex with men in China. AIDS Care 2020 Dec;32(12):1573-1580. [doi: 10.1080/09540121.2020.1742864] [Medline: 32188267]

30. Laporte A, Jourdan N, Bouvet E, Lamontagne F, Pillonel J, Desenclos J. Post-exposure prophylaxis after non-occupational HIV exposure: impact of recommendations on physicians' experiences and attitudes. AIDS 2002 Feb 15;16(3):397-405. [doi: 10.1097/00002030-200202150-00011] [Medline: 11834951]

31. Almeda J, Allepuz A, Simon B, Blasco JA, Esteve A, Casabona i Barbarà J. [Non-occupational post-exposure HIV prophylaxis. Knowledge and practices among physicians and groups with risk behavior]. Med Clin (Barc) 2003 Sep 20;121(9):321-326. [doi: 10.1016/s0025-7753(03)73937-2] [Medline: 14499067]

32. Blackstock OJ, Moore BA, Berkenblit GV, Calabrese SK, Cunningham CO, Fiellin DA, et al. A cross-sectional online survey of HIV pre-exposure prophylaxis adoption among primary care physicians. J Gen Intern Med 2017 Jan;32(1):62-70 [FREE Full text] [doi: 10.1007/s11606-016-3903-z] [Medline: 27778215]

33. Castel AD, Feaster DJ, Tang W, Willis S, Jordan H, Villamizar K, et al. Understanding HIV care provider attitudes regarding intentions to prescribe PrEP. J Acquir Immune Defic Syndr 2015 Dec 15;70(5):520-528 [FREE Full text] [doi: 10.1097/QAI.0000000000000780] [Medline: 26247895]

34. National HIV Curriculum: nonoccupational postexposure prophylaxis. 2020. URL: https://cdn.hiv.uw.edu/pdf/prevention/ nonoccupational-postexposure-prophylaxis/core-concept/all [accessed 2020-08-06]

35. The worry about privacy disclosure from specialized infectious disease hospitals. Sina. 2015. URL: http://henan.sina.com.cn/ news/hot/2015-06-15/detail-ifxczqar0905361-p3.shtml [accessed 2020-08-06] 
36. Post-exposure prophylaxis to prevent HIV infection: joint WHO/ILO guidelines on post-exposure prophylaxis (PEP) to prevent HIV infection. World Health Organization. 2007. URL: https://apps.who.int/iris/bitstream/handle/10665/43838/ 9789241596374 eng.pdf?sequence=1 [accessed 2020-08-06]

37. Marzel A, Heinrich H, Schilliger L, Fehr JS, Günthard HF, Kouyos R, et al. Prescription of postexposure prophylaxis for HIV-1 in the emergency room: correct transmission risk assessment remains challenging. J Acquir Immune Defic Syndr 2017 Apr 01;74(4):359-366. [doi: 10.1097/QAI.0000000000001265] [Medline: 27906766]

\author{
Abbreviations \\ aOR: adjusted odds ratio \\ CHERRIES: Checklist for Reporting Results of Internet E-Surveys \\ China CDC: Chinese Center for Disease Control and Prevention \\ IQR: interquartile range \\ MSM: men who have sex with men \\ nPEP: nonoccupational postexposure prophylaxis \\ oPEP: occupational postexposure prophylaxis \\ OR: odds ratio \\ PrEP: preexposure prophylaxis \\ UAI: unprotected anal intercourse \\ UNAIDS: Joint United Nations Program on HIV/AIDS \\ UVI: unprotected vaginal intercourse \\ WHO: World Health Organization
}

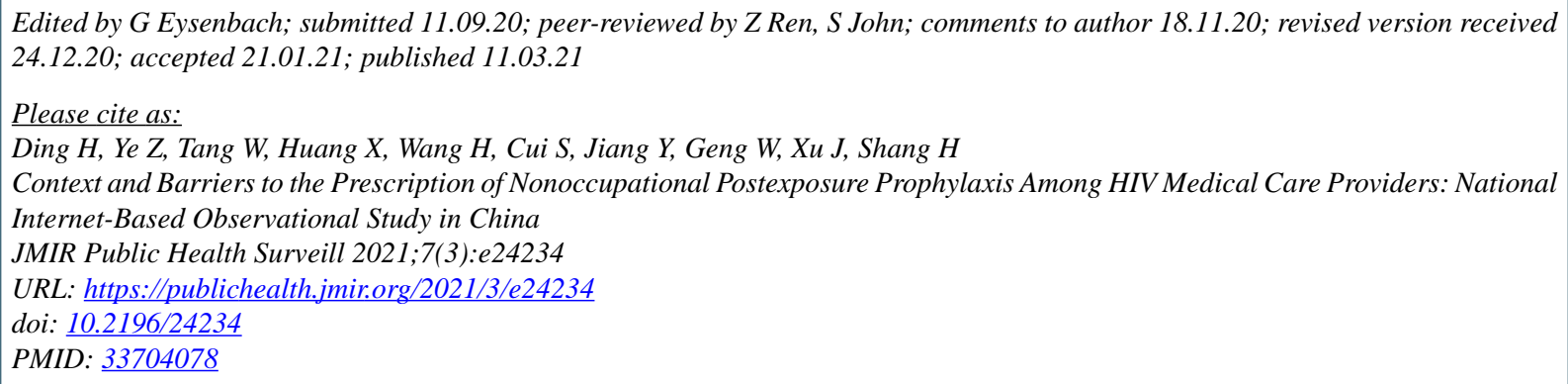

CHaibo Ding, Zehao Ye, Weiming Tang, Xiaojie Huang, Hui Wang, Sitong Cui, Yongjun Jiang, Wenqing Geng, Junjie Xu, Hong Shang. Originally published in JMIR Public Health and Surveillance (http://publichealth.jmir.org), 11.03.2021. This is an open-access article distributed under the terms of the Creative Commons Attribution License (https://creativecommons.org/licenses/by/4.0/), which permits unrestricted use, distribution, and reproduction in any medium, provided the original work, first published in JMIR Public Health and Surveillance, is properly cited. The complete bibliographic information, a link to the original publication on http://publichealth.jmir.org, as well as this copyright and license information must be included. 